\title{
Refractive index and electro-optic effect in compressive and tensile strained quantum wells
}

\author{
J. Pamulapati, J. P. Loehr, J. Singh, and P. K. Bhattacharya \\ Center for High-Frequency Microelectronics, Department of Electrical Engineering and Computer Science, \\ University of Michigan, Ann Arbor, Michigan 48109-2122
}

M. J. Ludowise

Hewlett-Packard Laboratories, 3500 Deer Creek Road, Palo Alto, California 94304

(Received 27 August 1990; accepted for publication 4 January 1991)

The effects of biaxial compressive and tensile strain on the excitonic resonances and associated changes in refractive index and electro-optic effect in quantum wells have been calculated and measured. Theoretical calculations include the important heavy-hole-light-hole band mixing effects. It is seen that the excitonic contributions dominate near the band edge. With increasing compressive strain the linear electro-optic effect is slightly increased, while the quadratic effect is greatly enhanced. The effects are reversed in quantum wells under tensile strain.

\section{INTRODUCTION}

The electro-optic properties of quantum wells are very different from that of single-layered materials. In a quantum well the valence band degeneracy is removed and this causes changes in the optical absorption. With the application of a transverse electric field, excitonic transitions do not disappear as in bulk materials but persist up to high fields. There are also changes in the energy eigenvalues of the quantum well and the coupling of electron and holes in the subband with the same quantum number are reduced. Theoretically, it has been shown, by considering only band-to-band transitions, that there is a large refractive index change for the TE mode in a multiple quantum-well (MQW) structure, resulting in a large electro-optic coefficient. ${ }^{1}$ Experimentally, it has been observed that the quadratic electro-optic effect becomes dominant as one approaches the excitonic edge. ${ }^{2}$

Biaxial strain in quantum wclls changes the energy separation and coupling between the heavy hole ( $\mathrm{HH})$ and light hole (LH) excitonic transitions. In the case of compressive strain, the $\mathrm{HH}$ absorption dominates the absorption edge, while in the case of tensile strain the light hole absorption dominates. Since the TE mode couples to the HH while the TM mode couples to the LH, this HH-LH splitting is expected to play an important role in determining the electro-optic properties. The effect of biaxial strain on the electro-optic effect in quantum wells has not been investigated in detail, both theoretically and experimentally, other than some preliminary experiments ${ }^{3}$ on InGaAs/GaAs MQW.

In this paper, we present calculated and measured data on the effect of biaxial strain on the excitonic resonances of quantum wells and consequently on their electro-optic properties. Measurements are carried out on molecularbeam epitaxial (MBE) $\mathrm{In}_{x} \mathrm{Ga}_{1}{ }_{x} \mathrm{As} / \mathrm{Al}_{0.2} \mathrm{Ga}_{0.8} \mathrm{As} \mathrm{MQW}$ structures and $\mathrm{In}_{0.53 \pm x} \mathrm{Ga}_{0.47 \mp x} \mathrm{As} / \mathrm{InGaAsP}$ quantum wells grown by metalorganic chemical vapor deposition (MOCVD). The phase shift is measured as a function of applied bias in waveguide electro-optic devices. It is seen that at fixed bias the phase shift increases with compressive strain and decreases with tensile strain. As will be evident later, this occurs primarily due to the changes in the LH-HH separation and not due to changes in the Stark effect in the strained quantum wells.

\section{ELECTRO-OPTIC PROPERTIES OF PSEUDOMORPHIC QUANTUM WELLS}

To model the optical properties of semiconductor quantum wells it is important to include the strong coupling between the heavy hole (HH, $\left.\left|\frac{3}{2}, \pm \frac{3}{2}\right\rangle\right)$, and light hole ( $L H,\left|\frac{3}{2}, \pm \frac{1}{2}\right\rangle$ ) states; the conduction band may be treated in the parabolic approximation. We account for the valence band coupling by using the $4 \times 4 \mathrm{k} \cdot \mathrm{p}$ Hamiltonian. ${ }^{4}$ The effect of strain is incorporated by including a splitting $\delta$ between the light and heavy hole diagonal elements. For $\mathrm{In}_{x} \mathrm{Ga}_{1-x}$ As grown on GaAs and $\operatorname{In}_{0.53 \pm x} \mathrm{Ga}_{0.47-x}$ As grown on InP it is given (in $\mathrm{eV}$ ) by $\delta=-5.966 \epsilon$, where the lattice mismatch $\epsilon$ is related to the excess In composition $x$ by $\epsilon=-0.07 x .^{5}$ This splitting reduces the offdiagonal mixing between the $\mathrm{HH}$ and $\mathrm{LH}$ states and changes the effective masses of the holes. In addition, the strain-induced splitting dramatically changes the difference between the light and heavy hole subband energies. We solve the Hamiltonian in the presence of strain and electric fields by using a finite difference numerical technique. ${ }^{6} \mathrm{We}$ also solve for the electron states in the presence of fields and compute the transition energies. We see that the electric field shifts both the light and heavy hole transition energies quadratically (the quantum confined Stark effect). In addition, we see that the strain strongly affects the zerofield transition energies for heavy and light holes. In the lattice matched case the light hole bandgap is slightly larger than that of the heavy hole because of the larger quantization energy associated with its lighter mass. The application of compressive strain, though, greatly increases this separation; applying tensile strain reduces the light hole energy below that of the heavy hole. The splitting strongly influences the optical properties because of the polarization dependence of the selection rules applying to optical transitions. TE mode ( $\hat{x}$ polarized) light couples three times more strongly to HH states than to LH states and TM mode ( $\hat{z}$ polarized) light couples only to the $\mathrm{LH}$ 


\begin{tabular}{lc}
\hline $200 \AA$ & $\mathrm{p}^{+} \mathrm{GaAs}$ \\
\hline $0.5 \mu \mathrm{m}$ & $\mathrm{p}^{+} \mathrm{Al}_{0.3} \mathrm{Ga}_{0.7} \mathrm{As}$ \\
\hline $0.5 \mu \mathrm{m}$ & $\mathrm{Al}_{0.2} \mathrm{Ga}_{0.8} \mathrm{As}$ \\
\hline $875 \AA$ & $\mathrm{In}_{x} \mathrm{Ga}_{1-x} \mathrm{As}-75 \AA(5$ Wells $)$ \\
& $\mathrm{Al}_{0.2} \mathrm{Ga}_{0.8} \mathrm{As} 100 \AA$ \\
\hline $0.5 \mu \mathrm{m}$ & $\mathrm{Al}_{0.2} \mathrm{Ga}_{0.8} \mathrm{As}$ \\
\hline $0.8 \mu \mathrm{m}$ & $\mathrm{n}^{+} \mathrm{Al}_{0.3} \mathrm{Ga}_{0.7} \mathrm{As}$ \\
\hline
\end{tabular}

(a)

\begin{tabular}{lc}
\hline $0.1 \mu \mathrm{m}$ & $\mathrm{p}^{+} \operatorname{In}_{0.53} \mathrm{Ga}_{0.47} \mathrm{As}$ \\
\hline $1.0 \mu \mathrm{m}$ & $\mathrm{p}^{+} \operatorname{InP}$ \\
\hline $0.5 \mu \mathrm{m}$ & $\mathrm{p}^{+} \operatorname{InGaAsP}(\lambda=1.1 \mu \mathrm{m})$ \\
\hline $0.1 \mu \mathrm{m}$ & $\operatorname{InGaAsP}(\lambda=1.3 \mu \mathrm{m})$ \\
\hline $360 \AA$ & $\operatorname{In}_{x} \mathrm{Ga}_{1-x} A \mathrm{As}-90 \AA(3$ Wells $)$ \\
& $\operatorname{InGaAsP}(\lambda=1.3 \mu \mathrm{m})-30 \AA$ \\
\hline $0.1 \mu \mathrm{m}$ & $\operatorname{InGaAsP}(\lambda=1.3 \mu \mathrm{m})$ \\
\hline $0.5 \mu \mathrm{m}$ & $\mathrm{n}^{+} \operatorname{InGaAsP}(\lambda=1.1 \mu \mathrm{m})$ \\
\hline $0.8 \mu \mathrm{m}$ & $\mathrm{n}^{+} \operatorname{InP}$ \\
\hline
\end{tabular}

FIG. 1. Schematics of (a) InGaAs/AlGaAs quantum wells grown by molecular-beam epitaxy, and (b) InGaAsP/InP quantum wells grown by mctalorganic chemical vapor deposition.

states; ${ }^{7}$ growth is done in the $z$ direction. Hence, the separation between $\mathrm{HH}$ and $\mathrm{LH}$ states will strongly affect the absorption spectra and the associated birefringence. After obtaining the bandstructure we calculate the excitonic binding energies and oscillator strengths via a nonvariational numerical technique. ${ }^{8}$

To determine the effect of strain and electric fields on the refractive index, we apply the Kramers-Kronig transform to the absorption spectra, yielding the result

$$
n\left(\omega_{0}\right)-1=\frac{c}{\pi} P \int_{0}^{\infty} \frac{\alpha(\omega) d \omega}{\omega^{2}-\omega_{0}^{2}},
$$

where $\omega_{0}$ is the frequency of the incident light. ${ }^{9}$ We find that the excitonic effects dominate the electro-optic effect when the input light energy is close to the band gap. In the results shown only the excitonic effects are included. Bandto-band effects are much smaller.

\section{RESULTS AND DISCUSSION}

Experiments were performed with $\mathrm{In}_{x} \mathrm{Ga}_{1-x} \mathrm{As} /$ $\mathrm{Al}_{0.2} \mathrm{Ga}_{0.8} \mathrm{As}(0 \leqslant x \leqslant 0.09)$ MQW $p-i-n$ structures grown by MBE to study the effect of compressive strain. $\mathrm{In}_{0.53 \pm x} \mathrm{Ga}_{0.47 \mp x} \mathrm{As} / \mathrm{InGaAsP}$ MQWs, grown by MOCVD, were used to measure the effect of compressive and tensile strain. Schematics of the two types of structures are shown in Figs. 1(a) and 1(b), respectively. The thickness of the MQW region is chosen such that it remains in the pseudomorphic regime. The effect of the strain should therefore be only on the well region of the MQW.
Ridge waveguides with contacts on the substrate and top $p^{+}$layers were fabricated using photolithography and ion-milling techniques. For modulation measurements 2-3 $\mathrm{mm}$ long waveguides were cleaved along the [011] and [01ㅣ crystallographic directions. The InGaAs/AlGaAs diodes have leakage currents of $100 \mathrm{nA}$ at $-10 \mathrm{~V}$ and reverse breakdown voltages of $\sim 20 \mathrm{~V}$ in the dark. The InGaAs/InGaAsP diodes have leakage currents of $100 \mu \mathrm{A}$ at $-4 \mathrm{~V}$ and reverse breakdown voltages of $\sim 6 \mathrm{~V}$ in the dark. The optical measurements were done with two sources: a Ti:sapphire laser with $\lambda=0.845-0.946 \mu \mathrm{m}$ for the InGaAs/AlGaAs system, and a Burleigh Color Center laser with $\lambda=1.48-1.67 \mu \mathrm{m}$ for the InGaAs/InGaAsP system. The laser light was end-fired on to the cleaved edge of the waveguides through an objective lens. The near field pattern at the output of the waveguide was focused on to a silicon or germanium detector through an analyzer. For electroabsorption measurements, the output, with the analyzer adjusted for maximum transmission, was recorded for different applied bias for input polarizations both parallel (TE) and perpendicular (TM) to the layers. For phase modulation measurements the waveguide was excited with a polarization oriented at $45^{\circ}$ to the direction of the applied electric field to equally excite both TE and TM modes in the guide.

We have fixed the energy difference between the excitation and the excitonic peak to be $\sim 90$ and $15 \mathrm{meV}$ for the $\mathrm{In}_{x} \mathrm{Ga}_{1-x} \mathrm{As} / \mathrm{Al}_{0.2} \mathrm{Ga}_{0.8} \mathrm{As}$ and the $\mathrm{In}_{0.53 \pm x} \mathrm{Ga}_{0.47 \mp x} \mathrm{As} / \mathrm{InGaAsP}$ systems, respectively. By

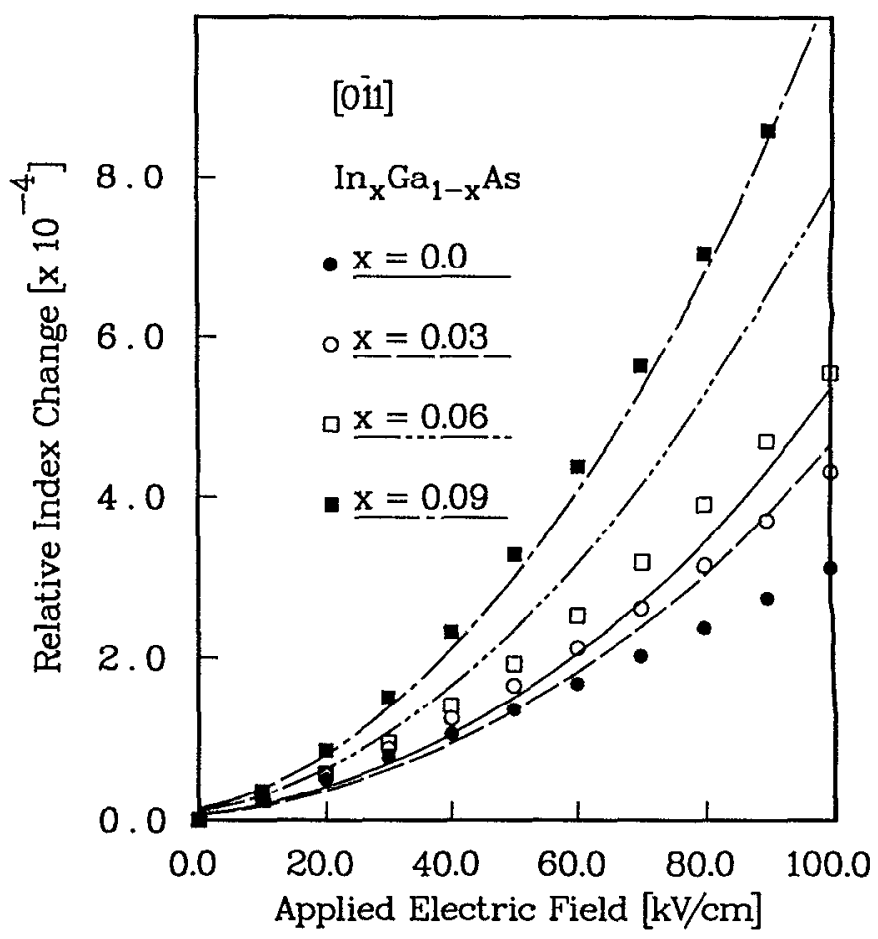

FIG. 2. Experimentally measured (points) and theoretically calculated (lines) values of $\Delta n$ as a function of applied electric field in the $\mathrm{In}_{x} \mathrm{Ga}_{1-x} \mathrm{Al} / \mathrm{Al}_{0.20} \mathrm{Ga}_{0.80} \mathrm{As} \mathrm{MQW}$ system. The different curves are for varying amounts of strain in the well. 
TABLE I. Measured linear and quadratic electro-optic coefficients in $\mathrm{In}_{x} \mathrm{Ga}_{1}-x \mathrm{As} / \mathrm{Al}_{0.2} \mathrm{Ga}_{0.8}$ As strained MQW.

\begin{tabular}{lcccc}
\hline \hline \multicolumn{1}{c}{$x$} & $\begin{array}{c}\lambda \\
(\mu \mathrm{m})\end{array}$ & $\begin{array}{c}\Delta E \\
(\mathrm{meV})\end{array}$ & $\begin{array}{c}r_{63} \\
\left(10^{-12} \mathrm{~m} / \mathrm{V}\right)\end{array}$ & $\begin{array}{c}R_{33}-R_{13} \\
\left(10^{-20} \mathrm{~m}^{2} / \mathrm{V}^{2}\right)\end{array}$ \\
\hline 0 & 0.8965 & 92 & 1.08 & 3.48 \\
0.03 & 0.9215 & 100 & 1.09 & 9.09 \\
0.06 & 0.9345 & 89 & 1.10 & 15.54 \\
0.09 & 0.9460 & 77 & 1.29 & 34.40 \\
\hline \hline
\end{tabular}

fixing this energy separation, we can extract the effect of strain on the electro-optic coefficients. The electro-optic coefficients $r_{63}$ and $\left(R_{33}-R_{13}\right)$ were obtained by comparing the measured phase changes with the calculated values according to: ${ }^{3}$

$$
\begin{aligned}
\Delta \Phi_{[1 \mathrm{i} 0]}= & \frac{\pi n_{\|}^{3} L}{\lambda}\left[ \pm \Gamma_{1} r_{63} \bar{E}_{j}+\Gamma_{2}\left(R_{33}-R_{13}\right) \bar{E}_{j}^{2}\right] \\
& +\Delta \Phi_{0}
\end{aligned}
$$

where $n_{\|}$is the average refractive index in the multilayer for polarization parallel to the layer, $\bar{E}_{j}$ is the average junction electric field, $\Gamma_{1}$ and $\Gamma_{2}$ are the overlap factors of the optical mode with the junction electric field and the square of the junction electric field, respectively, and $L$ is the length of the waveguides. The built-in birefringence in the MQW, $\Delta \Phi_{0}$ can be expressed as:

$$
\Delta \Phi_{0}=\left(\beta_{\mathrm{TE}}-\beta_{\mathrm{TM}}\right) L=\Delta n L\left(\frac{2 \pi}{\lambda}\right)
$$

where

$$
\Delta n=\left(n_{\|}-n_{1}\right) .
$$

The values of $\Gamma_{1}$ and $\Gamma_{2}$ in our samples are 0.12 and 0.15 , respectively. Variations of $\Gamma_{1}$ and $\Gamma_{2}$ with wavelength are assumed to be small and the junction electric field is assumed to be constant. The values of the linear and quadratic electrooptic coefficients obtained by fitting the data to Eq. (2) are given in Table I for the InGaAs/AlGaAs samples. The values obtained for the InGaAs/InGaAsP system are listed in Table II.

For both quantum-well systems, the data indicate an increase in the electrooptic coefficients with the use of compressive strain. The major change, though, is manifested in the quadratic electro-optic coefficient $R_{33}-R_{13}$. In addition, the data for the $\mathrm{In}_{0.45} \mathrm{Ga}_{0.55} \mathrm{As} / \mathrm{InGaAsP}$ system shows a decrease in the electro-optic coefficients.

We have theoretically formulated the changes in the refractive index due to strain and find that our experimen-

TABLE II. Measured linear and quadratic electro-optic coefficients in $\mathrm{In}_{0.53+x} \mathrm{Ga}_{0.47-x} \mathrm{As} / \mathrm{InGaAsP}$ strained MQW.

\begin{tabular}{cccc}
\hline \hline$x$ & $\begin{array}{c}\lambda \\
{[\mu \mathrm{m}]}\end{array}$ & $\begin{array}{c}r_{63} \\
{\left[10^{-12} \mathrm{~m} / \mathrm{V}\right]}\end{array}$ & $\begin{array}{c}R_{33}-R_{13} \\
{\left[10^{-20} \mathrm{~m}^{2} / \mathrm{V}^{2}\right]}\end{array}$ \\
\hline-0.08 & 1.584 & 2.83 & 146 \\
0 & 1.65 & 3.74 & 282 \\
0.07 & 1.67 & 4.10 & 389 \\
\hline
\end{tabular}

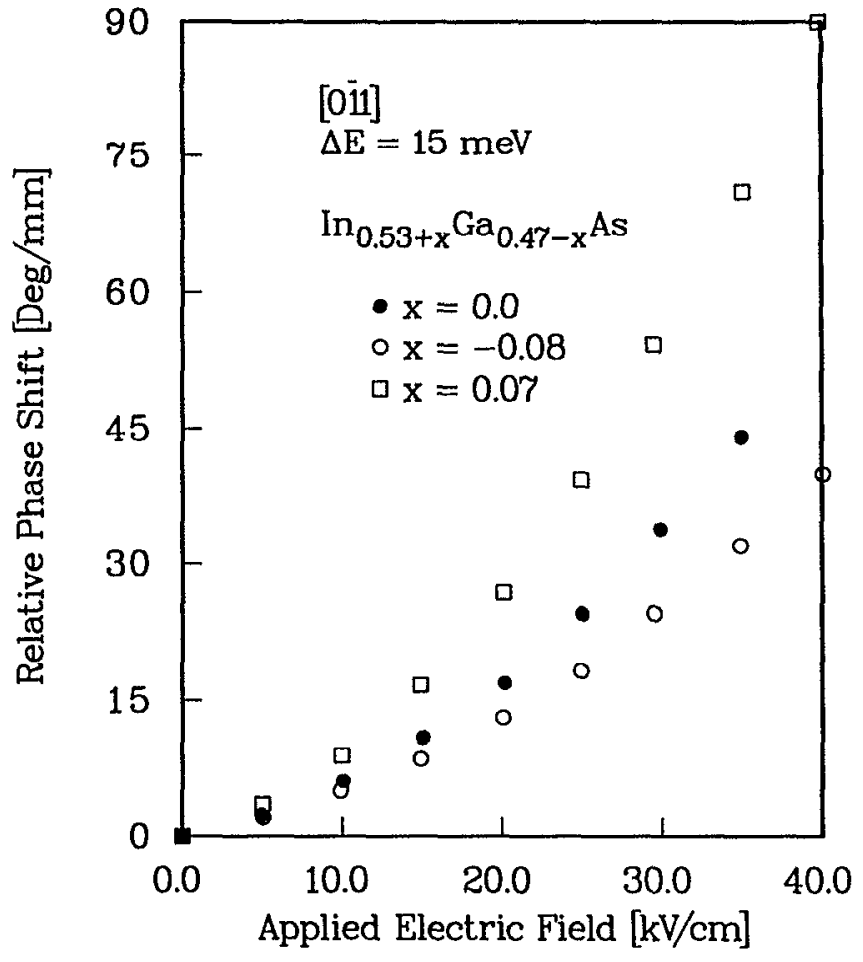

FIG. 3. Phase difference between Te- and TM-like modes for propagation in the [011] direction in the $\mathrm{In}_{0.53+{ }_{x}} \mathrm{Ga}_{0.47-x} \mathrm{As} / \mathrm{InGaAsP}$ system.

tal results are in agreement with both the calculated values and values obtained by others. ${ }^{10,11}$ Figure 2 shows the experimentally measured data and the theoretically calculated results for the InGaAs/AlGaAs system. The lines shown in Fig. 2 are obtained from a theoretical model, while the points are obtained from ${ }^{10}$

$$
\Delta n=-\frac{1}{2} n_{0}^{3}\left[r_{63} E+\left(R_{33}-R_{13}\right) E^{2}\right] .
$$

We feel that to the first order our theoretical model closely resembles the trend we see in the data. Figure 3 shows the measured phase change as a function of applied field for the InGaAs/InGaAsP systems. We do not show a theoretical fit in Fig. 3 because our model does not converge well when modeling the refractive indices when the incident light is so close to the band edge $(\sim 15 \mathrm{meV})$.

\section{CONCLUSION}

We report the effect of strain on the refractive index of InGaAs/A1GaAs and InGaAs/InGaAsP MQWs. The experimental data show that it is possible to slightly tailor the linear electro-optic coefficient, $r_{63}$, while drastically changing the quadratic electro-optic coefficient, $R_{33}-R_{13}$, with the use of compressive and tensile strain. The theoretical formalism shows good agreement with the experimental data.

\section{ACKNOWLEDGMENTS}

This work is supported by the Office of Naval Research under Grant No. N0001490-J-1831 and the Army Research Office (URI Program) under contract DAAL03- 
87-K-0007. The authors gratefully acknowledge the help and facilities provided by S. Gupta and other members of the Ultrafast Sciences Laboratories, University of Michigan, for some of the measurements. One of us (M.J.L.) wishes to acknowledge Bill Perez for expert assistance with epitaxial growth and characterization and John Turner for SIMS characterization.

${ }^{1}$ H. Yamamoto, M. Asada, and Y. Suematsu, Electron. Lett. 21, 579 (1985).

${ }^{2}$ M. Glick, D. Pavuna, and F. K. Reinhart, Electron. Lett. 23, 1235 (1987).
${ }^{3}$ U. Das, Y. Chen, P. K. Bhattacharya, and P. R. Berger, Appl. Phys. Lett. 53, 2129 (1988).

${ }^{4}$ J. M. Luttinger and W. Kohn, Phys. Rev. 97, 869 (1955).

${ }^{5}$ H. Kato, N. Iguchi, S. Chika, M. Nakayama, and N. Sano, J. Appl. Phys. 52, 588 (1986).

${ }^{6}$ M. Jaffe and J. Singh, J. Appl. Phys. 65, 338 (1989).

${ }^{7}$ S. C. Hong, M. Jaffe, and J. Singh, IEEE J. Quantum Electron. 23, 2181 (1987).

${ }^{8}$ J. P. Loehr and J. Singh, Phys. Rev. B 42, 7154 (1990).

${ }^{9}$ J. S. Weiner, D. A. B. Miller, and D. S. Chemla, Appl. Phys. Lett. 50, 842 (1987).

${ }^{10}$ J. E. Zucker, T. L. Hendrickson, and C. A. Burrus, Appl. Phys. Lett. 52, 945 (1988).

"J. E. Zucker, I. Bar-Joseph, B. I. Miller, U. Koren, and D. S. Chemla, Appl. Phys. Lett. 54, 10 (1989). 